\title{
Periorbital Necrotizing Fasciitis in a Young Woman with Anorexia Nervosa-the Management and Considerations of Cosmetic and Functional Outcomes of Fulminant Disease in a Critically Unwell Patient
}

\author{
Joshua Givorshner ${ }^{1 *}$ and Rafat Ghabrial ${ }^{2}$ \\ ${ }^{1}$ Department of Ophthalmology Registrar, The University of Sydney, Australia \\ ${ }^{2}$ Head of Department of Ophthalmology, Royal Prince Alfred Hospital, Australia
}

Submission: January 05, 2020; Published: January 13, 2020

*Corresponding author: Joshua Givorshner, Department of Ophthalmology Registrar, The University of Sydney, Australia

\begin{abstract}
Necrotizing fasciitis is a rapidly progressive and potentially deadly bacterial infection of the superficial fascia. The horrific and grossly debilitating nature of necrotizing fasciitis has led to an increased prominence of its reporting in mainstream media however the disease entity has not been historically well understood, classified or reported in medical literature. While capable of manifesting in numerous bodily locations following inoculation during trauma, the periorbital region is the most commonly involved facial site and presents unique challenges in treatment of the disease due to the unique anatomical and functional attributes of this area. This case report describes the diagnosis, surgical and medical management in a young woman with severe malnutrition due to anorexia nervosa.
\end{abstract}

Keywords: Necrotizing fasciitis; Infection; Oculoplastics; Periorbital; Ophthalmology; Reconstructive surgery; Immunocompromise(d)

\section{Introduction}

Necrotizing Fasciitis (NF) is a devastating rapidly progressive bacterial infection involving the superficial fascia leading to necrosis of overlying skin and systemic toxicity [1,2]. We present a case of periorbital NF in a chronically malnourished and unwell patient following facial trauma.

\section{Case Report}

A 36-year-old female presented to the emergency department following a mechanical fall with extensive left facial bruising extending to the neck and chest wall. The patient featured a complex medical history including severe anorexia nervosa. Pathology revealed significant electrolyte derangement, hypothermia, osteoporosis, bone marrow suppression and synthetic hepatic impairment. A clinical diagnosis of NF was made 6 days into her ICU admission following the development of anaesthetic, necrotic-appearing skin associated with purulent exudates from the palpebral fissure and a corresponding rise in the patient's White Cell Count (WCC) and C-Reactive Protein (CRP). Examination of the eye was unremarkable, with the exception of a subconjunctival hemorrhage and nasally oriented corneal dellen. Initial emergency debridement was undertaken as a joint operation between ophthalmology and ENT teams, stripping back necrotic periorbital tissues down to the lower cheek and performing a lateral tarsorrhaphy to prevent exposure keratopathy. ENT undertook further exploration and debridement down to the neck and submental region, leaving a VAC dressing insitu and harvesting multiple tissue samples for histopathological evaluation. Histology revealed marked suppurative inflammatory changes in the reticular dermis, skeletal muscle and subcutaneous fat in association with venous congestive changes and large regions of muscular and dermal necrosis (Figure 1). 


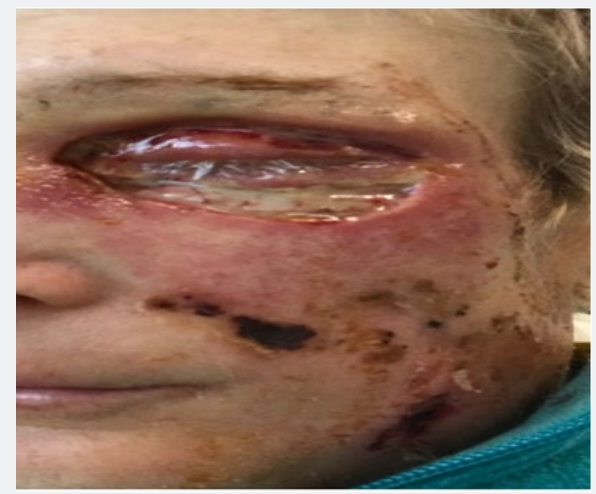

Figure 1: Post-operative appearance following initial operative debridement. Note the absence of upper and lower eyelid skin and tarsorrhaphy.

Pan-sensitive Streptococcus pyogenes and penicillinresistant Staphylococcus aureus (MSSA) were isolated from the excised tissues, while Pseudomonas species were isolated from the superficial left facial and eye swabs. Pending sensitivities, targeted antimicrobial therapy was instituted including the use of intravenous flucloxacillin, vancomycin, piperacillin-tazobactam and meropenem. Subsequent operative debridement and exploration was undertaken two days later, excising further nonviable periorbital tissues down to the buccal fat pad. Inspection of oral tissues was performed under direct laryngoscopy along with extension of tissue debridement from the neck area, noting that progression did not appear to track along fascial planes. Two months later, initial reconstruction surgery was performed.
After washout of the left cheek and periorbital wounds, a Split Skin Graft (SSG) was applied to the left cheek (from a left thigh donor site) and Full-Thickness Skin Graft (FTSG) was applied to the superior and inferior left eyelids (abdominal donor site). The left tarsorrhaphy was also revised. Six months following her initial presentation, horizontal lateral canthotomy and division of tarsorrhaphy was performed to allow for a wider palpebral fissure. Finally, nine months after the initial diagnosis of NF, debulking of the upper lid was performed in conjunction with suturing of trace remaining elevator fibers to the tarsal plate. Reconstruction of the lower eyelid with FTSG (donor site medial upper arm) was also performed to relax the cicatricial nature of the earlier procedures (Figure 2).

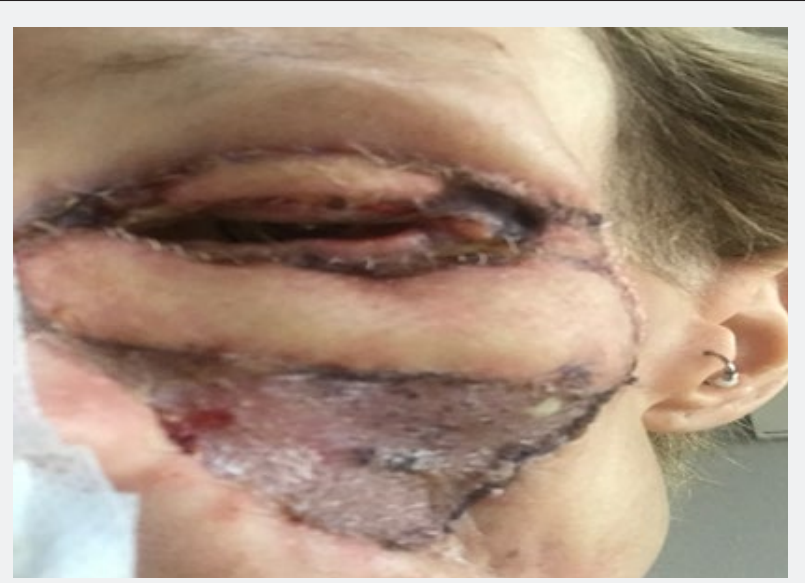

Figure 2: Appearance following initial reconstructive procedure demonstrating the bulk of the periorbital tissue and the narrow palpebral fissure.

Post-operative best corrected visual acuity was $6 / 9+2$ in the affected eye with $5-6 \mathrm{~mm}$ of lagophthalmos and complete pupillary exposure, allowing for good binocular vision without altered head posture. The patient remains able to use frontalis and orbicularis action to consciously moisten her cornea. The patient continued to use regular preservative-free lubricants to prevent exposure keratopathy. Periorbital NF is a rare rapidly progressing ophthalmic emergency that requires early recognition and operative debridement to prevent a fulminant course [1,3-5]. First described by Hippocrates in the fifth century B.C. as a complication of erysipelas [5], NF was then first properly described in modern literature by Confederate army surgeon Joseph Jones in 1871. Inconsistent nomenclature and inadequate linkage to sufficient bacteriological data led to confusion in the literature and a delay in the understanding of NF as a single disease entity [6]. Infection of the head and neck is uncommon in NF with only a 
small number of cases being described in literature since 1960. In the post-antibiotic era, the overall mortality of head and neck NF has been estimated at between 9 and 31\%, but its relatively frequent association with multi-system shock and permanent disfigurement render NF a particularly devastating and terrifying illness [3,7] (Figure 3).

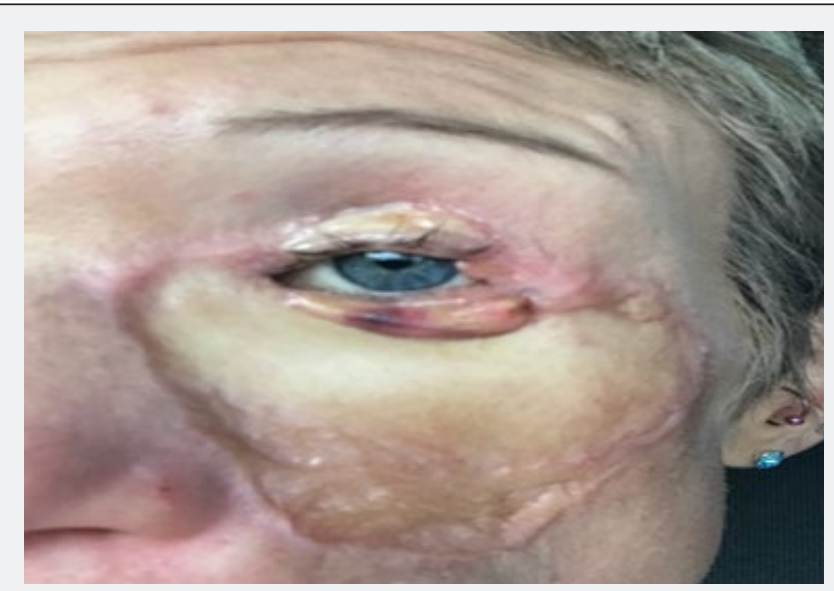

Figure 3: Appearance following final reconstruction and skin graft.

The most common trigger for periorbital NF is an antecedent injury leading to the breakdown of the skin barrier and concomitant inoculation of the causative organism(s) [6-8]. Swelling, pain, erythema, fever, haemorrhagic bullae, skin necrosis and crepitus are the most common clinical signs relevant to diagnosis $[2,4]$. Diabetes mellitus, immunosuppression, chronic renal impairment, pulmonary disease, recent surgery and traumatic injury have been identified as predisposing factors for NF; however, it is worthwhile noting that a large number of published cases remain idiopathic in nature [1,9-10]. Immunosuppression, age greater than 50 years, cirrhosis, congestive cardiac failure, gout and development of toxic shock syndrome (a late sign of infection) have been identified as independent risk factors most associated with increased mortality from NF $[1,2,11]$. Relative risk indicators have been identified, utilizing biochemical indicators such as white cell count, haemoglobin, CRP, serum creatinine, sodium and blood glucose to stratify NF severity in the acute setting [12].

Periorbital NF is divided into 4 classifications on the basis of microbiological culture. Type 1 (polymicrobial) infections are related to both anaerobic and aerobic (including facultative anaerobes such as Enterobacteriaciae and non-typable Streptococci) organisms most commonly manifesting on the abdomen, trunk and perineum. Type $1 \mathrm{NF}$ is the most common type to develop in patients with diabetes. Type 2 (monomicrobial) $\mathrm{NF}$ involves the extremities, cutaneous and muscular tissues; and features no clear underlying risk factors. Group-A betahaemolytic Stretococcus (S. pyogenes) and/or S. aureus are the most commonly implicated organisms in Type 2 disease, characterizing its strong association with toxic-shock syndrome. Type 3 infections are caused by Clostridium, Vibrio spp. and other gram-negative organisms, typically involving the abdominal wall and/or perineum. They are often rapidly progressive infections following salt-water injuries, seafood ingestion and penetrating traumas. Type $4 \mathrm{NF}$ describes fungal infections involving the extremities, most frequently occurring in immunosuppressed individuals $[1,9,10,13]$.

Our patient was a young, immunosuppressed, malnourished female. On hospital presentation she had severe electrolyte derangement (hypomagnesaemia, hypokalaemia and hyponatreamia), hypothermia, impaired hepatic synthetic function and coagulopathy (INR 2.0 with hypovitaminosis-K) and ischaemic digits. She developed severe facial Type $2 \mathrm{NF}$ (S. aureus and S. pyogenes) following a break in the periorbital skin overlying a large haematoma, initially caused by blunt mechanical trauma. No underlying fractures were identified on computed tomography imaging. Anaesthetic necrosis of the thin eyelid skin allowed the early clinical recognition and diagnosis of necrotizing fasciitis, nevertheless the infection was rapidly progressive in nature, resulting in severe permanent disfigurement despite prompt operative debridement and broad-spectrum antimicrobial cover.

This case affords some insights into the pathophysiology of $\mathrm{NF}$ and also presented challenging aspects in the consideration of reconstructive techniques, attempting to maximize both long-term functional stability of the eye in limiting exposure keratopathy whilst optimizing the patient's cosmesis.

Operative exploration and debridement in this patient demonstrated that this infection did not respect fascial planes, considered anomalous in the typical subcutaneous spread pattern of NF [14]. It is possible that traumatic disruption of subcutaneous compartments involving all skin and muscular layers, in conjunction with a large haematoma facilitated alternative pathways for the dispersion of infection in this patient. This is of particular importance to keep in mind in such cases where 
the musculoaponeurotic structures of the face and neck may be breached, allowing the spread of infection into the superior mediastinum [11].

\section{Discussion}

Periorbital NF is the most commonly involved facial site and behaves differently from NF elsewhere in that the highly vascularized orbicularis oculi acts as an effective barrier to prevent the spread of infection from the skin to the underlying periorbita, thereby retarding the spread of infection into the orbit. As in this case, necrosis of the thin eyelid skin occurs rapidly, facilitating early detection of the condition due to the obvious cosmetic abnormality on examination [1]. Given the fragile general health and constitution of the patient, decisions regarding the donor sites for reconstructive skin grafting were based purely on pragmatism, with cosmesis playing a clearly secondary consideration to what were emergent and life-saving surgeries. Gross malnutrition and immunosuppression meant that donor tissue was chosen to maximize graft viability, to facilitate adequate coverage of the relatively large areas left exposed following extensive surgical debridement and to maximize the chances of adequate postoperative healing in this setting. Subsequent procedures were required in this case to balance the cosmesis and function of the patients eyelids, her vision (and the initial requirement for a chinup posture to peer through her tight palpebral fissure,) and the necessity to prevent an exposure keratopathy in a patient with significant lagophthalmos secondary to an almost obliterated orbicularis.

\section{Conclusion}

The rapidly destructive nature of $\mathrm{NF}$ necessitates early recognition and diagnosis to minimize the risk of a fulminant course with high morbidity and mortality. Physicians must remain vigilant and mount a thorough clinical suspicion for $\mathrm{NF}$ in suitably vulnerable patients with clinical features suggestive of the disease such as severe pain, swelling, erythema, bullae and tactile anaesthesia. Urgent resuscitation, emergency surgical debridement, broad-spectrum IV antibiotics and other novel therapies such as hyperbaric oxygen must be implemented without delay to prevent the frequently debilitating, disfiguring and life-threatening sequelae of NF $[1,8,11,15]$. This case report demonstrated some of the complexities in managing periorbital
$\mathrm{NF}$ in the context of pre-existing medical comorbidities and highlighted the challenges in optimizing the functional and cosmetic outcomes of reconstructive surgery following extensive emergency debridements of neck and facial tissues.

\section{References}

1. Amrith S, Pai VH, Ling WW (2013) Periorbital necrotizing fasciitis-a review. Acta Ophthalmologica 91(7): 596-603.

2. Goh T, Goh LG, Ang CH, Wong CH (2014) Early diagnosis of necrotizing fasciitis. British Journal of Surgery 101(1): e119-e125.

3. Knudtson KJ, Gigantelli JW (1998) Necrotizing fasciitis of the eyelids and orbit. Archives of Ophthalmology 116(11): 1548-1549.

4. Lloyd RE (1987) Necrotizing fasciitis of the orbit. British Journal of Oral \& Maxillofacial Surgery 25(4): 323-327.

5. Pepe I, Lo Russo L, Cannone V, Giammanco A, Sorrentino F, et al. (2009) Necrotizing fasciitis of the face: a life-threatening condition. Aging Clin Exp Res 21(4-5): 358-362.

6. Carter PS, Banwell PE (2004) Necrotising fasciitis: a new management algorithm based on clinical classification. International wound journal 1(3): 189-198.

7. Shindo ML, Nalbone VP, Dougherty WR (1997) Necrotizing fasciitis of the face. Laryngoscope 107(8): 1071-1079.

8. Marchesi A, Marcelli S, Parodi PC, Perrotta RE, Riccio M, et al. (2017) Necrotizing Fasciitis in Aesthetic Surgery: A Review of the Literature. Aesthetic Plast Surg 41(2): 352-358.

9. Lancerotto L, Tocco I, Salmaso R, Vindigni V, Bassetto F (2012) Necrotizing fasciitis: Classification, diagnosis, and management. J Trauma Acute Care Surg 72(3): 560-566.

10. VanderMeulen H, Pernica JM, Roy M, Kam AJ (2017) A 10-Year Review of Necrotizing Fasciitis in the Pediatric Population: Delays to Diagnosis and Management. Clin Pediatr (Phila) 56(7): 627-633.

11. Schurr C, Burghartz M, Miethke T, Kesting M, Hoang N, et al. (2009) Management of facial necrotizing fasciitis. Eur Arch Otorhinolaryngol 266(3): 325-331.

12. Ali SS, Lateef $F(2016)$ Laboratory risk indicators for acute necrotizing fasciitis in the emergency setting. Journal of Acute Disease 5(2): 114116.

13. Sun XF, Xie T (2015) Management of Necrotizing Fasciitis and Its Surgical Aspects. Int J Low Extrem Wounds 14(4): 328-334.

14. Richir MC, Schreurs HH (2013) Facial Necrotizing Fasciitis. Surgical Infections 14(4): 428-429,

15. Thrane JF, Pikelis A, Ovesen T (2017) Hyperbaric oxygen may only be optional in head and neck necrotizing fasciitis: a retrospective analysis of 43 cases and review of the literature. Infect Dis (Lond) 49(11-12): 792-798. 
This work is licensed under Creative Commons Attribution 4.0 License DOI: 10.19080/JHNSS.2020.04.555631

\section{Your next submission with Juniper Publishers will reach you the below assets}

- Quality Editorial service

- Swift Peer Review

- Reprints availability

- E-prints Service

- Manuscript Podcast for convenient understanding

- Global attainment for your research

- Manuscript accessibility in different formats ( Pdf, E-pub, Full Text, Audio)

- Unceasing customer service

Track the below URL for one-step submission https://juniperpublishers.com/online-submission.php 